\title{
Fortalecimiento de las competencias investigativas en el contexto de la educación superior en Colombia'
}

\author{
María Inés Pérez Rocha
}

\begin{abstract}
Resumen
La educación superior debe emprender importantes transformaciones a fin de propiciar cambios significativos, que posibiliten la formación de profesionales competentes y lleven a la realización personal del educando, a partir del desarrollo, dominio y fortalecimiento de las competencias investigativas sobre la máxima para hacer hay que ser, fundamentado en un saber. Es importante que el docente estimule la investigación, mediante procesos de sensibilización, reflexión, experimentación, vivencia, análisis, crítica y razonamiento. A partir de la utilización de estrategias pedagógicas dinamizadoras centradas en el estudiante, que conlleven a la construcción significativa de conocimiento y el aprendizaje autónomo, integrando conocimientos, destrezas y actitudes.
\end{abstract}

Palabras clave: procesos educativos, competencias investigativas del saber, hacer y ser, estrategias pedagógicas. 


\title{
Strengthening of research skills in the higher education context in Colombia
}

\begin{abstract}
Higher education should undertake important transformations in order to promote significant changes, it enabling the training of proficient professionals and carry out the personal grow of the students, starting from the development, ownership and strengthening the investigative skills based on the maxim "for to do, must be being, based on knowledgement". It is important that the teachers encouraging the research, through different kind of processes like awareness, reflection, experimentation, experience, analysis, critical and reasoning. With using of dynamize teaching strategies focused on student, in order to meaningful construction of knowledge and independent learning, integrating knowledge, skills and attitudes.
\end{abstract}

Key words: Educational process, investigative skills from knowledge, doing and being, pedagogical strategies.

Recibido: 23 de febrero 2012

Aceptado: 25 de mayo 2012

\section{Introducción}

En la formación por competencias se integran tres aspectos fundamentales: el cognitivo que conduce a la apropiación, comprensión e interiorización del conocimiento; la puesta en práctica de las habilidades o destrezas que llevan a la aplicación del saber y el ser como aspecto más importante del proceso formativo dado el carácter actitudinal y comportamental. Ser competente es saber hacer y saber actuar con base en un conocimiento, expresado en valores y principios, con el propósito de que los estudiantes sean capaces de apropiarse de sus talentos, potencialidades y resuelvan problemas en un determinado contexto a partir de un aprendizaje significativo, autónomo y activo.

El desarrollo de competencias investigativas implica saber utilizar el conocimiento en forma adecuada, afianzando habilidades para observar, preguntar, argumentar, sistematizar, a fin de crear o gestionar el conocimiento, 
sobre la base del interés, la motivación hacia la investigación, el desarrollo de sus capacidades y la realización personal del estudiante.

En este sentido, el docente ha de estar en capacidad de cuestionarse y revisar su quehacer, replantear su didáctica, estrategias pedagógicas, actitudes, valores y sus propias competencias investigativas; un profesor con convicción que trascienda lo eminentemente académico, que forme para la vida, propicie el reconocimiento de las potencialidades de sus educandos y propenda por el avance y competitividad de las instituciones.

De acuerdo con lo anterior, es necesario el análisis y aplicación de las estrategias pedagógicas tales como: el aprendizaje cooperativo, el método de proyectos, el aprendizaje basado en problemas, la utilización del mapa conceptual, el estudio de casos, los seminarios, la simulación didáctica, el ensayo y el taller entre otros. La utilización de estas estrategias conlleva al fortalecimiento de las competencias investigativas que permiten fomentar el espíritu investigativo. A partir de la integración de lo que se sabe con lo que se hace y se es, mediante procesos vivenciales, cambio de metodologías pasivas y contextos de aprendizaje.

\section{Desafios de la educación superior en el contexto nacional}

La educación superior se ve enfrentada a grandes retos como son: la adaptación al medio en permanente cambio; el aumento de la cobertura con calidad y equidad; incorporación de nuevas tecnologías a los procesos educativos; gestión del conocimiento; la formación investigativa con capacidad para aportar soluciones a las necesidades y problemáticas del contexto. Una formación que integre las competencias del ser, saber y saber hacer; procesos de enseñanza aprendizaje que permitan estructurar currículos para la vida; desarrollo de potencialidades y satisfacción de las necesidades del ser humano; una educación incluyente con calidad, que permita la construcción de bases sólidas para la vida y el trabajo; mejoramiento de la capacidad de gestión con una mayor calidad organizativa y administrativa; sistemas de financiación eficientes para alcanzar los objetivos misionales de la Universidad; procesos que desarrollen la innovación y la creatividad del estudiante, entre otras.

En concordancia con lo anterior, «la finalidad de la enseñanza universitaria sigue siendo el transmitir los conocimientos y desarrollar las competencias de alto nivel. Tradicionalmente, las finalidades asignadas a la enseñanza universitaria giraban alrededor de un doble objetivo: asegurar el dominio de conocimientos sólidos y recientes en un campo científico y/o profesional dado, desarrollar en los estudiantes la capacidad de pensar por sí mismos y poner en perspectiva crítica los saberes enseñados. Sin embargo, esta misión podría parecer hoy imposible» (Gómez y Alzate, 2009: 19). 
Fortalecimiento de las competencias investigativas en el contexto de la educación superior en Colombia, artículo original producto de la investigación

De acuerdo con lo anterior, algunos de los aspectos sobre los cuales la universidad necesita una reflexión y revisión son: la deficiente calidad académica de algunas instituciones; la presencia de esquemas pedagógicos dogmáticos y autoritarios; dificultad en la implementación de nuevas tecnologías de información y comunicación; consolidación de una comunidad académica a partir del trabajo interdisciplinario en donde se tomen decisiones conjuntas; la escasa participación en el desarrollo regional y nacional; la generación de conocimiento adecuado para enfrentar problemáticas sociales; el tipo de contratación de los docentes; gestión administrativa, que tenga en cuenta la experiencia, formación y perfil del profesorado; formación pedagógica de los docentes; la masificación y mercantilización de la educación superior; ausencia de incentivos que generen una mayor motivación, disposición y apropiación del ejercicio docente.

Las respuestas de la educación superior frente a las exigencias de la sociedad, del mundo y su capacidad para cambiar y solucionar los propios problemas, dependen del saber que las instituciones logren acumular sobre sí mismas y sobre sus relaciones con el entorno, el conocimiento y la cultura en general. En consecuencia, la educación superior necesita conformar un cuerpo de conocimientos y una masa crítica que, además de dar cuenta de su propio quehacer, promueva la generación de teorías, proyectos y propuestas de solución más pertinentes, que contribuyan a la formulación de políticas y a la toma de decisiones, tanto por parte del Estado, como de las mismas instituciones.

Las instituciones de educación superior han de comprometerse a formar profesionales competentes, que se desempeñen exitosamente en el mercado laboral, a partir de una formación integral fundamentada en valores, actitudes y comportamientos que incentiven la pasión por la investigación; por lo cual es necesario el fortalecimiento de la misma, entendida como un proceso de búsqueda, generación y construcción de conocimiento, sistemático, controlado, reflexivo y crítico, basado en el deseo de indagación, comprobación, comprensión y cambio de la realidad, la cual permite explicaciones lógicas, coherentes, innovadoras y creativas.

Rojas, define la investigación «como un proceso de construcción de conocimiento, de indagación, de interrogación requiere, así mismo, la construcción de sujetos que indaguen y se interroguen, que se expongan y se arriesguen en esa irracionalidad insegura que es la ciencia. En el caso del estudiante universitario, la cultura de la investigación es una necesidad prioritaria para adquirir conocimiento de todos los saberes que desconoce con respecto al problema y a la formulación de hipótesis para llegar a las conclusiones precisas y darles solución» (2005: 86).

\section{Fortalecimiento de las competencias investigativas}

La noción de competencia fue introducida por Noam Chomsky para explicar las competencias lingüísticas, lo que ha permitido la construcción de una 
pedagogía eficaz para el desarrollo del aprendizaje de la lengua en el ámbito educativo. Para Angarita el concepto de competencia « trata de un conocimiento especializado o de carácter específico; es un conocimiento implícito en la práctica o de carácter no declarativo; deriva solo parcialmente de un proceso de aprendizaje, aún cuando requiere de la experiencia social y cultural» (2000: 29).

Es importante tener en cuenta que el desarrollo de las competencias se ve condicionado en un principio, por un sistema de vivencias, conocimientos y creencias que se desarrollan en los primeros años de vida de la persona, influenciados por aspectos sociales y culturales, que definen la estructura de su personalidad e influyen directamente sobre sus comportamientos y actitudes.

\section{Para los autores Cázares y Cuevas}

En la noción de competencia se integran cuatro saberes básicos: el saber por sí mismo, como conocimiento base y explicativo que considera la comprensión; el saber hacer, como la puesta en juego de habilidades basadas en los conocimientos; el saber ser, como la parte más compleja por sus implicaciones de carácter actitudinal e incluso valorar, y el saber transferir, como la posibilidad de trascender el contexto inmediato, para actuar y adaptarse a nuevas situaciones o transformarlas. Ser competente es saber hacer y saber actuar entendiendo lo que se hace, comprendiendo cómo se actúa, asumiendo de manera responsable las implicaciones y consecuencias de las acciones realizadas y transformando los contextos a favor del bienestar humano (2008: 17).

Sobre la base de los argumentos anteriormente enunciados, es necesaria una formación integral que tenga en cuenta las competencias investigativas del ser, el hacer y el conocer. Aprender a ser involucra el desarrollo de valores como el compromiso, el respeto, la tolerancia, la solidaridad, la participación, la autonomía, el amor propio, la coherencia, el trabajo en equipo y la sana convivencia, entre otros. En el hacer se observa, analiza, interpreta, deduce, comprende y se construye conocimientos, se realiza la investigación a partir de una realidad concreta. Y en el saber, se adquieren conocimientos acerca de la investigación científica, cómo se formulan problemas, hipótesis, redacción de objetivos viables y medibles, trabajo de campo, técnicas e instrumentos, análisis e interpretación de la información, que permita realizar investigación de calidad.

En este sentido, la competencia además de ser un saber hacer, es un saber haciendo, soportado en múltiples vivencias y conocimientos que se van adquiriendo en el transcurso de la vida; es la utilización flexible e inteligente de los conocimientos que se tienen, lo que nos hace competentes frente a esas tareas específicas. En otras palabras, quien es competente lo es para una actividad determinada. Una competencia no puede entenderse como capacidad 
Fortalecimiento de las competencias investigativas en el contexto de la educación superior en Colombia, artículo original producto de la investigación

intelectual o mental, requiere de la acción en un contexto y del ser a nivel actitudinal y comportamental.

De igual modo, la competencia es la capacidad de integrar lo que se hace con lo que se sabe y se es; ser competente es saber hacer y saber actuar desde lo que se es como ser humano, con convicción de cuanto se hace y asumiendo de manera ética y comprometida las implicaciones de sus actos acordes con un contexto determinado.

\section{Para Le Boterf las competencias.}

Pueden ser consideradas como el resultado de tres factores: el saber proceder que supone saber combinar y movilizar los recursos pertinentes (conocimientos, saber hacer, redes...); el querer proceder que se refiere a la motivación y a la implicación personal del individuo; el poder proceder que remite a la existencia de un contexto, de una organización del trabajo, de condiciones sociales que otorgan posibilidad y legitimidad en la toma de responsabilidad y riesgo del individuo.

En el modelo sistémico sobre las competencias, la competencia es un proceso. Así, ser competente es saber proceder, eso implica ser capaz de movilizar las diferentes funciones de un sistema compuesto por diversos recursos, sistemas de razonamiento, conocimientos, evaluaciones, capacidades, etc. Sus componentes son:

Las entradas están compuestas de las situaciones y tareas profesionales a las que el sujeto está enfrentado en su quehacer profesional y/o laboral. La gama de acciones es muy variada porque son variados los empleos, los métodos y los contextos profesionales y de trabajo.

Un conjunto de funciones pueden ser puestas en juego por el sujeto. Éstas pueden conducir al saber proceder constitutivo de la competencia. La elaboración de representaciones operacionales permitirá seleccionar o combinar los conocimientos y las operaciones pertinentes y concernientes con la tarea a realizar. La consideración de la imagen de sí mismo es fundamental porque la confianza en sí mismo o la apreciación de las propias potencialidades son factores decisivos para poner en marcha la dinámica de la competencia. La activación de los saberes memorizados: conocimientos teóricos, procedimientos, experiencias, etc. Como recursos movilizables, la activación del saber hacer cognitivo permite operaciones de inferencia produciendo informaciones nuevas a partir de las disponible y determinadas situaciones. La construcción de conocimientos tanto por los procesos de formación como por los procesos de trabajo. 
Las prácticas profesionales y las actuaciones son las salidas del modelo. Están directamente relacionadas con la situación. Son estas prácticas y actuaciones las que deben ser consideradas en última instancia en un proceso de evaluación de competencias. La decisión de que una práctica profesional es competente no es tomada por una o unas determinadas selecciones de recursos. Así, las diferentes combinaciones posibles e individuales dan diferentes posibilidades de actividades profesionales competentes (Le Boterf, 1998: 50).

En este contexto, es importante fortalecer las competencias investigativas, integrando la investigación formativa y la investigación propiamente dicha, lo que permite fomentar el espíritu investigativo, a través de la participación en investigaciones que dirijan los docentes, el intercambio de ideas, la disposición hacia la investigación, la capacidad de asombro, indagación y construcción, que forme en y desde la libertad en donde prime el respeto, la tolerancia y la capacidad de aceptación del potencial del otro, conformando equipos de trabajo donde se ponga al servicio los talentos y las potencialidades de cada persona de tal forma que se logre un trabajo en equipo con calidad.

Es relevante cuestionarse frente al grado de correspondencia entre las competencias investigativas y las desarrolladas en la academia en el trabajo docente; toda vez que el desarrollo de habilidades, destrezas, aptitudes y conocimientos, en un determinado campo del saber y en un contexto social determinado conforman el conjunto de dominios indispensables para el fortalecimiento de las competencias investigativas en el estudiante.

La investigación es un quehacer rico en experiencias valiosas, cognoscitivas, intelectuales y prácticas significativas para la formación del estudiante, quien amplia sus conocimientos, habilidades y destrezas, plantea interrogantes a partir del qué, el cómo y el para qué; por esta razón, si el estudiante demuestra ser competente y tiene la capacidad para resolver una situación determinada, esto depende del conocimiento, del aprendizaje derivado de la experiencia y de su actitud para enfrentar la situación. Por tal motivo, es fundamental definir y desarrollar las competencias investigativas a nivel del saber, hacer y ser:

La competencia del saber: hace referencia al tipo de competencias cognitivas, que llevan a identificar un problema, búsqueda, selección y sistematización de información, comprensión, análisis, síntesis, evaluación, inducción y deducción del conocimiento; corresponde al manejo conceptual que los estudiantes tienen sobre el saber especifico y su aplicación en contextos sociales, la acción de conocer es esencial para afianzar el pensamiento científico, buscar soluciones, tener un pensamiento autónomo y crítico. 
Fortalecimiento de las competencias investigativas en el contexto de la educación superior en Colombia, artículo original producto de la investigación

Las competencias cognoscitivas son:

- Dominio de los conceptos de ciencia, técnica y tecnología.

- Conocimiento sobre la epistemología de la investigación.

- Conocimiento del método de investigación cualitativa y cuantitativa.

- Comprensión del proceso de investigación.

- Dominio de los tipos de investigación.

- Entendimiento de los problemas de investigación.

- Identificación de la función de los objetivos de la investigación.

- Identificación de los marcos de referencia y comprensión en torno de su elaboración.

- Identificación de la función del marco teórico en investigación.

- Identificación de como se formula y comprueba una hipótesis.

- Comprensión de la función del diseño metodológico en investigación.

- Establecimiento de la diferencia entre población y muestra.

- Conocimiento de las técnicas e instrumentos de recolección de información.

- Conocimiento del análisis, interpretación e identificación del para qué de los resultados de la investigación.

- Dominio de las herramientas estadísticas para el procesamiento de la información.

- Identificación de nuevas tecnologías de información y comunicación.

- Conocimientos que le permiten proponer soluciones.

- Identificación de los contenidos del informe final de investigación. 
- Dominio de la presentación de informes de investigación.

- Identificación de pensamiento independiente, disciplinado, creativo y reflexivo.

- Dominio en el manejo de las condiciones intelectuales asociadas con la atención, memoria y concentración.

- Conocimiento de la búsqueda, selección y comprensión de la información.

- Dominio de interpretación, argumentación y proposición.

- Conocimiento de técnicas e instrumentos de recolección de la información.

- Comprensión de la lógica y razonamiento inductivo y deductivo.

- Conocimiento interdisciplinario y multidisciplinario.

- Dominio de significados en torno de un campo del saber.

- Formación con visión científica y tecnológica del mundo.

- Formación para el análisis y síntesis.

- Entendimiento de descripciones y explicaciones.

- Identificación de resúmenes y síntesis.

- Comprensión de la repetición, inferencia y discernimiento.

- Identificación de la deducción, inducción o generalización.

En este orden de ideas, las personas aprenden de forma relevante cuando adquieren conocimientos que consideran útiles e importantes para su vida, desarrollan la capacidad de pensamiento independiente, disciplinado, creativo y reflexivo que lo lleve a entender las múltiples perspectivas del conocimiento, de su saber, comprensión y descubrimiento.

Aprender para conocer supone, en primer término, aprender a aprender, ejercitando la atención, la memoria y el pensamiento, a partir de la combinación del método inductivo y deductivo. La competencia del saber integra la experiencia, perspectivas, cultura, intereses, necesidades, aprendizaje previo, que implica los factores cognitivos apoyados en una 
Fortalecimiento de las competencias investigativas en el contexto de la educación superior en Colombia, artículo original producto de la investigación

selección subjetiva de datos e información disponible. Aprender es adquirir significados, es entrelazar una red de relaciones lógicas entre información que tiene sentido en el campo del saber y estos a su vez agrupan significados.

La competencia del saber hacer: el estudiante se motiva y aprende más cuando se le presentan problemas reales, siempre y cuando se propicie un clima de confianza y libertad capaz de potenciar el deseo de aprender. El aprendizaje es más efectivo cuando se tienen en cuenta las diferencias en el desarrollo físico, intelectual, emocional y social del estudiante, además sus interacciones y relaciones sociales influyen ampliamente en su desempeño; el educando aprende de manera relevante cuando el conocimiento adquiere significado o sentido para alcanzar un propósito en la vida; la participación en prácticas sociales constituye un aspecto fundamental de su actuación. En este orden de ideas, las competencias del saber hacer son entre otras:

- Capacidad para aplicar las teorías epistemológicas en la investigación.

- Habilidad para aplicar el método científico.

- Capacidad para aplicar el método cuantitativo y cualitativo.

- Capacidad para utilizar las líneas de investigación con actitud propositiva.

- Capacidad para seleccionar el tipo de investigación, métodos y técnicas.

- Habilidad para delimitar el tema de investigación.

- Capacidad para formular el problema de investigación.

- Destreza en la elaboración del estado del arte en la investigación.

- Capacidad para formular los objetivos de la investigación.

- Habilidad para realizar la justificación de la investigación.

- Capacidad para formular y comprobar hipótesis de investigación.

- Destreza en la elaboración de los marcos de referencia.

- Capacidad para efectuar búsquedas y actualización bibliográfica.

- Habilidad para elaborar el marco teórico que fundamenta la investigación. 
- Capacidad para operacionalizar variables.

- Capacidad para aplicar el diseño metodológico en la investigación.

- Capacidad para aplicar las técnicas e instrumentos de recolección de datos.

- Capacidad para identificar la unidad de análisis.

- Habilidad para aplicar las herramientas estadísticas.

- Capacidad para aplicar las técnicas e instrumentos de recolección de datos.

- Habilidad para elaborar conclusiones y recomendaciones.

- Capacidad de aplicación de los conocimientos.

- Capacidad de organización y planificación de la investigación.

- Capacidad para elaborar resúmenes y síntesis.

- Destreza en la creación de imágenes mentales y mapas conceptuales.

- Capacidad para analizar un informe de investigación.

- Capacidad para leer y escribir con claridad en forma eficaz.

- Capacidad para redactar una monografía, un ensayo, un trabajo científico.

- Destreza para la elaboración y redacción de textos, por ejemplo un artículo.

- Habilidad para redactar el resumen, el informe de avance o el final.

- Capacidad para hablar en forma clara, argumentada y convincente.

- Habilidad para manejar el computador y los recursos digitales.

- Habilidad para buscar y analizar la información.

- Capacidad para resolver problemas y encontrar soluciones prácticas.

- Destreza para la toma de decisiones acertadas bien razonadas y socialmente responsables.

- Habilidad para identificar oportunidades. 
Fortalecimiento de las competencias investigativas en el contexto de la educación superior en Colombia, artículo original producto de la investigación

- Destreza para movilizar y conseguir recursos.

- Manejo de las tecnologías de la información y de la comunicación.

- Capacidad para diseñar y desarrollar proyectos conjuntamente.

- Capacidad para elaborar el análisis, comparación y síntesis.

- Habilidad para coordinar y participar en reuniones de investigadores.

- Habilidad para el trabajo en grupo y actitud de aprendizaje.

- Capacidad para afrontar contextos cambiantes y complejos.

- Capacidad para seleccionar las publicaciones.

- Habilidad para aplicar con rigor científico los conocimientos.

- Capacidad para omitir vicios de redacción.

- Capacidad para jerarquizar, integrar y recordar datos.

- Capacidad para dar significado a la información con recursos semánticos o sintácticos.

- Capacidad para hacer preguntas y utilizar material de referencia.

- Aplicación de estrategias de asimilación de la información y retención.

- Capacidad para gestionar su propio aprendizaje.

- Destreza en el pensamiento critico, creativo e innovador.

- Habilidad para observar, registrar y elaborar notas de campo.

De este modo, se contribuye a la formación del estudiante para hacer frente a una cantidad de situaciones y problemas, que conlleven una experiencia formativa y una praxis social.

Competencia del ser: en el aprendizaje se desarrollan actitudes fundamentales para el crecimiento personal tales como la comprensión, la tolerancia, la empatía, la solidaridad, la cooperación, el respeto por sí mismo y por el otro, la valoración y el amor propio, la generosidad, la motivación, el querer hacer, el adecuarse y adaptarse a las diversidad de contextos, la capacidad para expresar emociones, la tolerancia a las frustraciones, las aspiraciones, sueños 
y esperanzas en la búsqueda de un futuro mejor; la disposición y confianza hacia el aprendizaje; la capacidad para superar obstáculos; la capacidad de asombro, de curiosidad y la dignidad, así como el reconocimiento de sus potencialidades y habilidades. En concordancia con lo anterior, las competencias del saber ser son:

- Capacidad para desarrollar en forma comprometida la investigación.

- Disposición positiva, gusto e interés por la investigación.

- Habilidad para percibir la investigación como un proyecto de vida.

- Capacidad para expresar ideas, sentimientos y emociones.

- Habilidad para trabajar en equipo.

- Capacidad de crítica y autocrítica.

- Habilidad interpersonal de cooperación y solidaridad.

- Capacidad para comunicarse asertivamente.

- Habilidad para solicitar reformulaciones y aclaraciones.

- Capacidad para respetar las normas ético-morales.

- Habilidad para trabajar en equipo interdisciplinario.

- Capacidad para respetar la diversidad y la multiculturalidad.

- $\quad$ Capacidad para ejercer liderazgo.

- Habilidad para trabajar en forma responsable y comprometida.

- Capacidad para fortalecer la autonomía y confianza en si mismo.

- Destreza para aportar su talento y desarrollar sus potencialidades.

- Capacidad para adaptarse al cambio.

- Capacidad para generar relaciones de confianza y respeto mutuo.

- Capacidad para trabajar con calidad, esfuerzo y compromiso.

- Ser consciente de la dimensión ética de sus actuaciones. 
Fortalecimiento de las competencias investigativas en el contexto de la educación superior en Colombia, artículo original producto de la investigación

- Madurez emocional para la comprensión de sí mismo y del otro.

- Sensibilidad y pensamiento crítico y creativo.

- Habilidad para establecer prioridades, programar el tiempo, disponer de recursos.

- Habilidad para evitar conflictos interpersonales, cooperar y motivar a otros.

- Capacidad para negociar y conciliar.

- Capacidad para ser tolerante y aprender a convivir.

En este sentido, recobra mayor importancia la capacidad de comunicarse asertivamente, de trabajar en equipo y resolver los conflictos, ponerse en el lugar del otro, entender sus emociones, posiciones y sentimientos, esto implica una formación para la convivencia democrática, la cooperación y la solidaridad, así como la participación activa y consciente. Por otra parte, el descubrimiento del otro, implica descubrirse a si mismo mediante la educación tomada como vía para alcanzar su realización personal y profesional, así como una actitud reflexiva ante el ejercicio de su libertad de los comportamientos éticos, la autonomía personal, la autocrítica y el ejercicio de sus valores.

\section{El papel del docente en la formación por competencias}

Para potencializar las competencias descritas del saber, del hacer y del ser, se requiere de un docente con convicción y compromiso; capaz de transcender lo académico, que se preocupe por la formación de sus educandos no solo cognitivamente, sino en competencias personales, interpersonales y axiológicas; que eleve la formación profesional y fortalezca su crecimiento personal; que amplíe el horizonte académico, a partir de la investigación social, que aporte conocimientos y experiencia para lograr cambios profundos y soluciones pertinentes; un docente inquieto y audaz en proceso permanente de formación pedagógica.

La actitud que el docente tenga hacia la investigación ejerce un papel fundamental en el espíritu investigador del educando; es pertinente que genere preguntas y cuestionamientos, que le apasione la investigación y reconozca la labor realizada, que no teja relaciones de asimilación pasiva y de dependencia con el saber, que sea creativo y conduzca los proyectos de investigación mediante el uso de métodos apropiados según el objeto y la teoría adoptados; que construya trayectoria investigando y articule a la investigación los semilleros, que forme y consolide redes de investigación y 
se interese por conocer las expectativas de sus estudiantes, sin desconocer que cada estudiante es único en la medida que ha experimentado una serie de factores vivenciales que influyen en su capacidad, motivación, expectativas y competencias.

De acuerdo con lo anterior, la investigación formativa en la educación superior ha de abordar el problema de la relación docencia-investigación, toda vez que requiere del análisis de las estrategias pedagógicas que utiliza el docente para el fortalecimiento de las competencias investigativas, lo cual implica el dominio del proceso de enseñanza y aprendizaje, principalmente basado en el ser.

Siguiendo esta idea, «La investigación formativa, vinculada a la enseñanza y al trabajo pedagógico, si bien es una problemática que se asocia preferentemente con la educación superior; en general se acepta que esta es una cuestión extensiva a todo el ámbito educativo. Muchos afirman que este conflicto es el mismo, pero en otro contexto diferente, que se da entre investigación y docencia, entre los paradigmas cuantitativos y cualitativos, y aún entre conocimiento y pedagogía» (Cerda, 2007: 59).

También hay que tener en cuenta que las competencias investigativas no han ocupado un lugar preponderante en los planes de estudio; lo que se pretende es que el estudiante adquiera un conjunto de conocimientos que le permitan la observación, interpretación, análisis, desarrollo del pensamiento complejo y crítico para el diseño, ejecución y evaluación de proyectos de investigación.

De otra parte, la función del docente en el fortalecimiento de las competencias investigativas es fundamental puesto que él constituye un ejemplo de proyecto de vida en el ámbito profesional, social y laboral; promueve la expresión de sentimientos y actitudes fundamentadas en valores; un pensamiento independiente, disciplinado, creativo, no dogmático, no repetitivo y no solo de transmisión de conocimientos; un cambio sustancial en la forma de enseñar y evaluar, lo cual supone un aprendizaje significativo de los aspectos que conciernen a la investigación. Lo anterior da lugar a la apertura de un espacio donde el estudiante elabore escritos, ensayos, artículos; en este aspecto, es clave que el docente comparta sus expectativas, aspiraciones y criterios para realizar la investigación.

De igual forma, se requiere de un docente que fortalezca la autoestima de los estudiantes resaltando sus aspectos positivos en los procesos de aprendizaje, dado que estos reaccionan de acuerdo con la percepción del docente, generando un clima de confianza y aceptación mutua, empatía y estimulo del aprendizaje por competencias, mediante procesos de experimentación y vivencia, el cambio de metodologías pasivas, unidireccionales a 
Fortalecimiento de las competencias investigativas en el contexto de la educación superior en Colombia, artículo original producto de la investigación

metodologías centradas en el estudiante, en el aprendizaje autónomo, activo y significativo, en el cual la enseñanza provoque, acompañe, oriente y cuestione el aprendizaje de los estudiantes. La enseñanza no es una simple habilidad, sino una compleja actividad cultural profundamente condicionada por hábitos y creencias. Se requiere cambiar la cultura para crear nuevos contextos de aprendizaje, que exigen nuevas estrategias de aprendizaje que incluyan la investigación y la acción, promoviendo un mayor énfasis en los resultados y en la gestión del conocimiento.

La necesidad de integrar el aprender a conocer, a hacer, a ser, da mayor protagonismo al estudiante en su formación; de igual modo, es imprescindible organizar los procesos de enseñanza y aprendizaje en función del desarrollo de estas competencias; son cambios que se producen en una sociedad del conocimiento, mediante la utilización de nuevas tecnologías de información y comunicación, con una serie de competencias intelectuales, metodológicas y técnicas con el propósito de facilitar los procesos de formación y capacitación de los investigadores.

En este orden de ideas, el profesor ejerce una clara influencia sobre el estudiante por su claridad conceptual, el dominio del proceso de investigación, la coherencia en su actuar, y por ello a de constituirse en un ejemplo de buenas prácticas intelectuales de indagación y actuación; sin perder de vista los aspectos personales de acuerdo con la ética, respeto por las diferencias, creatividad, innovación, motivación y compromiso para el desarrollo de la investigación. Los docentes deben enseñar mediante su testimonio a partir de sus experiencias y desarrollar competencias investigativas de manera eficaz de acuerdo a los contextos y a las situaciones.

\section{Estrategias pedagógicas dinamizadoras para el fortalecimiento de las competencias investigativas.}

En el desarrollo de estas competencias se requiere de una pedagogía que aporte sentido al acto educativo, al proceso de formación investigativa que conlleve a la construcción de su aprendizaje, posibilite la integración de conocimientos, habilidades, destrezas y actitudes. Las estrategias pedagógicas son un aspecto relevante para el fortalecimiento de las competencias investigativas.

Las estrategias pedagógicas constituyen los escenarios de los procesos de formación y de interacción de la enseñanza aprendizaje, donde se fortalecen conocimientos, valores, prácticas, procedimientos y problemas propios del campo de formación. Son las actividades u operaciones mentales que el estudiante desarrolla para optimizar su aprendizaje, tienen una clara intencionalidad y requieren de un plan de acción para que el estudiante adquiera el conocimiento. 
Para Medina (2004), las estrategias de enseñanza se basan en un conjunto de decisiones programadas que principalmente llevan al estudiante a adquirir conocimientos o habilidades, teniendo en cuenta el tipo de materiales, su organización y las actividades que deben desarrollarse, también surgen en función de los valores y las actitudes que se pretenden formar.

La estrategia pedagógica es el proceso, a través del cual el docente define procedimientos, cursos de acción, toma decisiones e integra operaciones cognitivas, habilidades, actitudes y valores en el aprendizaje significativo, de tal forma que el estudiante adquiera los elementos necesarios para el cumplimiento de un objetivo o tarea de manera eficaz en un contexto determinado.

Dentro de las estrategias pedagógicas encontramos:

Semilleros de investigación: Una de las estrategias para lograr lo enunciado anteriormente son los semilleros de investigación, que involucran al estudiante en los proyectos de investigación, los cuales deben ser autónomos, flexibles, participativos y cuyo propósito fundamental sea contribuir a la formación integral del estudiante, aprender de sus docentes, aprender haciendo e investigando.

Calderón, define a los semilleros de investigación como:

Una de las acciones emprendidas para fortalecer la investigación formativa iniciativa mediante la cual se desarrollan actividades de formación, conversatorios y proyectos. Los semilleros se conciben como un espacio de reconocimiento, donde estudiantes, egresados y docentes de las diferentes disciplinas convergen con un interés común: aprender a investigar. Deben ser un escenario para abordar la formación de investigadores desde la base para el desarrollo de competencias básicas de lectura, escritura, comunicación y metodológicas, en un ambiente que motive al individuo a involucrarse en el ejercicio de la investigación, que desarrollen su creatividad y su capacidad para asociarse y trabajar en equipo (2006: 48).

En este orden de ideas, el semillero forma estudiantes capaces de transformar y crear nuevos conocimientos o tomar decisiones, a través de procesos de investigación y el análisis e interpretación de la realidad utilizando las diversas tecnologías de información y comunicación; el aprendizaje por descubrimiento; cultivo de la actitud científica; sentido y razón de ser del proceso investigativo, al plantear soluciones a necesidades o problemáticas. Así mismo, que los futuros profesionales adquieran las habilidades y conocimientos necesarios, a través de la investigación formativa, el trabajo en grupo que les permita la construcción de comunidad académica; el incentivo a la creatividad, el análisis, el espíritu crítico; potencializar sus 
Fortalecimiento de las competencias investigativas en el contexto de la educación superior en Colombia, artículo original producto de la investigación

atributos y talentos; el creer en sí mismos, lo cual facilite la creación de su propio conocimiento a través de la apropiación y valoración de sus talentos y potencialidades.

El aprendizaje cooperativo: este se desarrolla en pequeños grupos de trabajo, a través de actividades didácticas orientadas a facilitar el aprendizaje en el aula, los estudiantes intercambian ideas, opiniones, experiencias, sentimientos, analizan, discuten, se organizan, se solidarizan, aprenden a intercambiar de acuerdo con sus percepciones; fortalecen la capacidad de escucha, la comunicación asertiva, el aprender a negociar, a conciliar, a entrar en consenso y discenso, ejercer adecuadamente el liderazgo, fomentar la creatividad para integrar a los estudiantes lo que pretende es centrar el aprendizaje en ellos.

El aprender a trabajar en equipo es una competencia profesional que se debe ejercitar en el proceso de enseñanza y aprendizaje; ello fortalece la habilidad para trabajar eficazmente con otras personas; el estudiante aprende cuando es sujeto activo del aprendizaje. Con el aprendizaje cooperativo se genera un sentido del nos, un sentido de pertenencia al grupo, se autorregulan los procesos centrados en la tarea y en los resultados.

\section{El método por proyectos: según Cerda}

Un proyecto es de hecho una investigación en profundidad de un tema o problema especifico que por su pertinencia y relevancia amerita estudiarse. La actividad implica la participación y la actividad de un grupo de estudiantes y es una experiencia que trasciende los estrechos contornos académicos de una clase. Los propósitos de un proyecto no son sólo buscar respuestas o dar soluciones, sino también desarrollar las competencias cognitivas y socio-afectivas de los estudiantes, particularmente si éste hace parte de la organización curricular y los componentes integrales del pedagógico (2007: 209).

En la experiencia del proyecto pedagógico se reproducen todas las fases y formas de trabajo de un proyecto de investigación científica. Los motivos para la realización de un proyecto son: satisfacer una necesidad relevante de un grupo humano, una institución o persona; o en su defecto superar los obstáculos que impidan la satisfacción de esa necesidad; resolver un problema relevante y complejo, dentro de su contexto y de un sistema determinado de valores; introducir y orientar un proceso de cambio que se considera necesario y deseable de acuerdo con ciertos valores, efectuar transformaciones y mejoras a una actividad o servicio.

El método de proyectos se fundamenta en el aprendizaje significativo, el cual requiere de la organización de sus contenidos de tal forma que el aprendizaje 
se base en un hecho real o problema, y el planteamiento de hipótesis que le permitan la construcción de conocimientos, de reflexión, análisis y síntesis dirigidos a la solución de un problema. Este método requiere una participación activa del estudiante en el planteamiento, diseño, realización y aplicación de la propuesta; requiere así mismo, de revisión bibliográfica, lecturas previas, hacer demostraciones, retroalimentar en forma oportuna, evaluación continua, fundamentación teórica y metodológica, trabajo en equipo y trabajo individual, que aporte al cumplimiento del objetivo, aspectos que influyen en la motivación del estudiante de aprender a conocer, aprender a hacer y aprender a ser. Esta estrategia pedagógica, además de desarrollar las competencias investigativas, también forma en habilidades, actitudes y valores como el compromiso, la responsabilidad, la tolerancia, la aceptación, el respeto, la cooperación, la solidaridad, autonomía, valoración, entre otros.

El aprendizaje basado en problemas, (ABP) propone la utilización de situaciones problemáticas, próximas a la vida real, que despierten el interés del estudiante y sea un estimulo para el aprendizaje.

Para Prieto «representa un enfoque ciertamente innovador que en los últimos ha cobrado un gran auge al ofrecer a los estudiantes escenarios profesionales reales que les permiten adquirir y poner en práctica, en un proceso de construcción personal y descubrimiento, las competencias necesarias para resolver situaciones problemicas a las que tendría que hacer frente en el ámbito profesional elegido» (2008:91).

En este contexto, se destaca por ser un aprendizaje basado en la investigación, la cual se deriva de estrategias previas a la lectura y buenos procesos de observación científica, a partir de preguntas como: ¿qué creemos que sabemos sobre el tema?, ¿qué necesitamos averiguar?, ¿qué esperamos aprender?, y por transferir el aprendizaje a la vida, fuera del aula, es decir, en el mundo social y cultural del que hacen parte los estudiantes.

El aprendizaje basado en problemas es una estrategia pedagógica fundamentada en el principio de identificación de problemas como punto de partida, para la adquisición e integración de nuevos conocimientos. Es un aprendizaje centrado en el discente y se produce en pequeños grupos, los docentes son facilitadores del proceso; los problemas son el eje fundamental de organización y estímulo para el aprendizaje; este se convierte en autodirigido; promueve, además, el aprendizaje integrado al unir el qué con el cómo y el para qué se aprende.

Sus fases o pasos son la presentación del problema, escenario del problema, aclaración de terminología, identificación de factores, generación de hipótesis, identificación de lagunas del conocimiento o necesidades de aprendizaje, facilitación del acceso a la información necesaria, resolución del problema o identificación de problemas nuevos. 
Fortalecimiento de las competencias investigativas en el contexto de la educación superior en Colombia, artículo original producto de la investigación

El mapa conceptual. La capacidad para elaborar mapas conceptuales esta muy relacionada con las habilidades del pensamiento abstracto, es una estrategia que permite medir el aprendizaje significativo, es necesaria la comprensión significativa de las relaciones entre los conceptos, reconocer la relevancia del contenido e integrar el conocimiento.

«El mapa es una representación esencialmente cognitiva y lógica, necesariamente coherente visual del conocimiento sobre un argumento preciso, pero con contornos flexibles (que tiene relaciones abiertas o relaciones abiertas o latentes con cualquier otro argumento) es principalmente conceptual y en alguna medida factual» (Hernández, 2007:52).

Contiene tres elementos fundamentales: proposición: consta de dos o más términos conceptuales unidos por palabras enlace para formar una unidad semántica; el concepto: es una regularidad en los acontecimientos o en los objetos que se designa mediante algún término y las palabras enlace son las palabras que sirven para unir los conceptos y señalar el tipo de relación existente entre ambos.

Desde la perspectiva más amplia de la teoría general de la educación, el mapa conceptual concuerda con un modelo educativo: centrado en el estudiante y no en el profesor, quien atiende al desarrollo de destrezas y no se conforma sólo con la repetición memorística de la información, que pretende el desarrollo armónico de todas las dimensiones de la persona y no solamente las intelectuales.

El estudio de casos: "Del latín casus, que significa suceso, acontecimiento, consiste en el análisis de una situación real, experiencia simulada, que tiene la finalidad de que el o los sujetos que reflexionan al respecto sean capaces de analizar, a partir de estas, otras situaciones reales, propias o ajenas a la experiencia real, de quien las discute» (Ferreiro, 2006: 109).

Es una estrategia de aprendizaje activo que tiene un primer momento de actividad individual y otros de trabajo en equipo y en grupo. El caso es un informe escrito, breve y conciso sobre una situación real y concreta, cercana a todos de una u otra forma, que despierta interés y provoca reflexión por estar redactado en forma de situación problema. El análisis de casos, además de desarrollar una toma de conciencia (meta cognición) sobre un tema objeto de estudio, estimula la posible aplicación práctica de lo analizado a una situación cercana y propia (transferencia).

Algunas de sus ventajas son las siguientes: propicia la lectura y el análisis de textos, entrena la escucha activa, estimula la comunicación interpersonal, la expresión oral y la argumentación, confronta nuevas situaciones problema para darle solución, permite la reflexión individual y en grupo, el diálogo y 
la discusión grupal, desarrolla el pensamiento estratégico y la capacidad de acción, entrena en la toma de decisiones; su valor pedagógico se relaciona con la reflexión que provoca (enseñar a pensar) y en su valor potencial para educar en valores.

En algunas oportunidades el caso puede ser una situación o circunstancia que ocupa un lugar en un momento determinado, pero que puede cambiar si se modifica la circunstancia. De igual manera, se puede constituir en un acontecimiento, que inicialmente es un suceso o un hecho particular, pero que posteriormente puede servir para caracterizar una realidad cotidiana más general. Es también un incidente, algo que ocurre durante el desarrollo de un asunto, que sin ser parte esencial, impide el desenvolvimiento normal de una acción. La tarea de estudio de casos frente a estos, es describirlos, caracterizarlos y ayudar a que se constituyan en un ejemplo particular de una categoría y de un estilo.

El seminario: según Ferreiro (2006), el término seminario, del latín seminarius, semillero, recuerda su primera finalidad: fuente de ideas necesarias para conocer y comprender la realidad y estar en condiciones de transformarla. En la didáctica contemporánea el seminario es una forma de organización del proceso de aprendizaje y enseñanza, que pone énfasis en el intercambio de ideas, criterios, opiniones, puntos de vista, dudas e interrogantes acerca de un tema seleccionado, entre otras cosas por ser complejo de comprender y controversial por su naturaleza, y sus implicaciones sociales.

Los seminarios constituyen una estrategia pedagógica con la finalidad de reunir, mediante el esfuerzo de todos los participantes, los elementos necesarios para construir una posición frente al tema objeto de estudio; constituyen asimismo un entrenamiento intelectual, individual y grupal, dirigido a esclarecer y a adoptar una posición en relación con un asunto álgido. En otras palabras, el seminario como estrategia reúne a estudiantes (previamente preparados cual expertos en las diversas aristas del tema), para que presenten sus ideas y discutan entre todos sus relaciones e implicaciones, de modo tal que lleguen a conclusiones, dado el objetivo del programa de la asignatura.

El seminario es una forma de organizar el proceso de aprendizaje con énfasis en la teoría. Es aquella que conduce a conocer integralmente, desde todas sus perspectivas, un proceso o fenómeno, con el fin de comprenderlo en esencia y estar en condiciones de actuar satisfactoriamente, sin repetir errores, con economía de esfuerzos y recursos pero, sobre todo, creativamente, a nivel de nuestro tiempo y condiciones.

El ensayo: es una composición literaria, es un escrito relativamente breve, producto de un ejercicio que implica la recolección de la información, su discernimiento, profundización, síntesis y sobre todo, la apreciación que el 
Fortalecimiento de las competencias investigativas en el contexto de la educación superior en Colombia, artículo original producto de la investigación

autor expresa, de manera particular, frente a ella. Redactar consiste en poner por escrito un pensamiento, una opinión, entre otros, aunque no todo tipo de escrito es el apropiado dentro del mundo académico. Al escrito académico lo llamamos ensayo; consta de cuatro partes:

El título, es un enunciado que encabeza el texto y que sirve de pretexto para indicar, orientar o soslayar el tema central del ensayo; el planteamiento o tesis, es recomendable que se la presente directamente, de manera sencilla y precisa. Ahora bien, si el escrito está basado en la profundización de un problema determinado, éste deberá exponerse o delimitarse abiertamente; ha de indicar si de lo que se trata es descubrir, probar, o refutar una hipótesis; proponer una nueva conjetura o simplemente examinar algunas observaciones hechas sobre el tópico en cuestión.

No sobra advertir que la tesis siempre tiene que hacer referencia a la temática tratada; la sustentación o argumentación, la ausencia de argumentos deshace la naturaleza del ensayo; la sustentación se soporta entonces en explicaciones, ejemplos, casos, y en todo tipo de justificaciones, que permitan al escritor bien comprobar o bien rechazar la proposición estudiada, los métodos utilizados y las estrategias empleadas en su estudio; así mismo, los datos y descripciones, la explicación y la interpretación de los datos obtenidos.

El Taller: es una estrategia pedagógica innovadora en la cual, mediante un conjunto de actividades, los sujetos aprenden en equipo para realizar y obtener, entre todos, algo concreto y significativo; es decir, es una forma de organización de la enseñanza que le permite a los participantes recrear y/o crear la realidad que les interesa, a partir del criterio epistemológico de concebir la ciencia y también el arte como un conjunto de conocimientos y actividades no resueltos totalmente, pero factibles de perfeccionarse y construirse entre todos.

Esta estrategia es recomendable utilizarla en temas prácticos, así como el seminario en los teóricos. Dos son las vías para conocer y comprender el mundo circundante, la realidad: la vía sensorial (órganos sensoriales) y la vía racional (pensamiento). Actualmente se ha reconocido que cada uno aporta datos imprescindibles para comprender y, a partir de ello, actuar de modo crítico y creativo.

El taller es un espacio para el desarrollo de vivencias emocionales, que conjuntamente con las racionales, forman parte de la realidad, lo que favorece de modo eficaz el aprendizaje significativo. Así mismo, permite que el estudiante trabaje plenamente, desarrollando tanto el cerebro derecho, responsable de lo socio-afectivo y espiritual, como el cerebro izquierdo, que es lógico, cuantitativo y secuencial.

Los talleres tienen en común los siguientes elementos didácticos: la creación de un ambiente socio-afectivo, en el que la comunicación horizontal y 
asertiva entre todos los participantes favorece el desarrollo de la imaginación creativa y reflexiva, además permite a los participantes expresar lo que piensan y creen acerca de la realidad con la emotividad que el sujeto y las relaciones interpersonales implican. De igual modo, la selección de las estrategias pedagógicas que faciliten, el logro de los objetivos planteados y orientar a los estudiantes para que puedan, por sí mismos y en equipo, realizar cabalmente la tarea planteada, reflexionar, vivenciar experiencias, hacer crítica constructiva y evaluar el aprendizaje.

Simulación didáctica: es una forma de organizar el proceso de aprendizaje y enseñanza grupal. Didácticamente, la simulación consiste en la recreación de una muestra selectiva e intencional de algún aspecto de la realidad física o social. Es la representación simplificada de un hecho, suceso o fenómeno que se requiere conocer y comprender en esencia y, más aún, en algunas ocasiones desarrollar determinadas habilidades al respecto.

Existen diferentes tipos de simulaciones didácticas, pero todas implican la activa y emotiva participación del sujeto que aprende, en una experiencia de aprendizaje que le va a proporcionar vivencias muy positivas en la construcción, bien de una noción teórica, bien de una habilidad relacionada con el saber hacer.

Es relevante aclarar que la simulación didáctica es un medio y nunca un fin. Es una estrategia que favorece la interiorización de aspectos sustantivos de la realidad que se aprehende, lo cual favorece extraordinariamente los procesos de sentido y significado, metacognición y transferencia, trascendentales para lograr aprendizajes significativos. Así mismo, el utilizarla permite la espontaneidad, la imaginación y la creatividad, posibilitando el aprendizaje placentero.

Las estrategia de simulación requiere ante todo un maestro mediador en el sentido estricto del concepto, con una actitud de cambio favorable, ya sea repetir fiel o creativamente la estrategia de un autor reconocido, o bien para crear él mismo una estrategia que pueda resultar igualmente eficaz pedagógicamente. Así, que son mediadores que no ocupan el centro del escenario sino, preparan muy bien la situación de aprendizaje, con posibles escenarios alternativos, las actuaciones de los estudiantes y dejando siempre un amplio rango de libertad de los sujetos participantes, el maestro se dedica a observar desde lejos y toma apuntes necesarios para la etapa de reflexión.

De acuerdo con lo anterior, las estrategias pedagógicas permiten la organización de los procesos de enseñanza aprendizaje, para el logro de los objetivos propuestos; la posibilidad de aprender de una forma diferente, a partir de la reflexión, análisis, la critica y el intercambio de experiencias; una participación activa del estudiante a través de un cambio fundamental de 
Fortalecimiento de las competencias investigativas en el contexto de la educación superior en Colombia, artículo original producto de la investigación

roles, siendo el mayor protagonista el estudiante; una experiencia cognitiva y vivencial, que le proporciona aprendizajes significativos; la construcción consciente y activa del aprendizaje; se afianzan actitudes, valores teniendo un impacto en la vida personal y social; desarrolla el aprendizaje cooperativo, habilidades para trabajar en equipo y generan claramente un mayor desarrollo de las competencias investigativas del ser, saber y hacer.

\section{Referencias bibliográficas}

Amaya, J. y E. Prado. 2003. Estrategias de aprendizaje para universitarios. México: Trillas.

Angarita, T. 2000. Los educadores del tercer milenio y la evaluación por competencias. Bogotá: Líto FOCET.

Calderón, M. 2006. Estado del subsistema de investigaciones. Bogotá: Universidad Distrital Francisco José de Caldas.

Caballero, P., M. Prada, E. Vera y J. Ramírez. 2007. Políticas y prácticas pedagógicas: las competencias en TIC en educación. Bogotá: Universidad Pedagógica Nacional.

Cázares, L. y J. Cuevas. 2008. Planeación y evaluación basadas en competencias. Fundamentos y prácticas para el desarrollo de competencias. México: Trillas.

Cerda, H. 2007. La investigación formativa en el aula. Bogotá: Investigar Magisterio.

Cortés, J., A. González, J. Huepe y otros. 2005. Semillero de investigación de Derechos Humanos. Avances en investigación formativa. Serie Documentos. Bogotá: Universidad del Rosario.

Díaz, F. y G. Hernández. 2007. Estrategias docentes para el aprendizaje significativo. México: McGraw Hill.

Escribano, A. y A. del Valle. 2008. El aprendizaje basado en problemas. Una propuesta metodológica en educación Ssperior. Madrid: Narcea.

Ferreiro, R. 2009. Nuevas alternativas de aprender y enseñar. Aprendizaje cooperativo. México: Trillas.

Ferreyra, H. y G. Peretti. 2006. Diseñar y gestionar una educación auténtica. Buenos Aires: Noveduc.

Flórez, R. 2005. Pedagogía del conocimiento. Bogotá: McGraw Hill. 
García, C. 2003. Los semilleros de investigación, hacia la reflexión pedagógica en la Educación Superior. Medellín: Biogénesis.

Gómez, M. y M. Alzate. 2009. Enseñanza y didáctica universitaria. Bogotá: ECOE.

Hernández, V. 2007. Mapas conceptuales. México: Alfaomega.

Le Boterf,G. 1998. Como gestionar la calidad de la formación. Barcelona: Aedipe.

Machado, E. y N. Montes. 2004. «Aprendizaje basado en la solución de tareas (ABST): contribución para la formación y desarrollo de habilidades investigativas en cursos posgraduados». Metodología de la investigación pedagógica y Universidad de Camagüey, Revista Iberoamericana de Educación.

Martínez, E. y M. Vargas. 2002. La investigación sobre la educación superior en Colombia. Un estado del arte. Bogotá: ICFES.

Maldonado, M. 2006. Competencias: método y genealogía. Bogotá: Ecoe.

Medina, A. 2004. Didáctica de las ciencias sociales. Madrid: Prentice Hall.

Ontonia, J. y A. Gómez. 2005. Potenciar la capacidad de aprender y pensar. Madrid: Narcea.

Orozco, C. 1993. Pedagogía de la educación superior. Bogotá: Universidad de los Andes.

Ossa, J. 2005. «Educar es enseñar a indagar la investigación como proceso de formación». Educaçao. Vol: XXVII:57: 525 - 533.

Ossa, J. 2008. El docente universitario y la formación investigativa. Memorias primer seminario interinstitucional sobre formación del profesorado para la educación superior. Facultad de Educación. Medellín: Grupo CHHES - Universidad de Antioquia. Vol.8 No.3.

Prieto, L. 2008. La enseñanza universitaria centrada en el aprendizaje. Estrategias útiles para el profesorado. Barcelona: Octaedro.

Restrepo, B. 2005. «Conceptos y aplicaciones de la investigación formativa, y criterios para evaluar la investigación científica en sentido estricto». Ubicado en http:// desarrollo.ut.edu.co/tolima/hermesoft/portal/home_1/rec/arc_6674.pdf

Rojas, M. 2005. Investigar la investigación. Ibagué: CERES.

Sarmiento, A., L. Tovar y C. Alam. 2001. «Educación, compromiso de todos». por Bogotá: Corpo-educación. 


\section{María Inés Pérez Rocha}

Fortalecimiento de las competencias investigativas en el contexto de la educación superior en Colombia, artículo original producto de la investigación

Stenhouse, L. 2004. La investigación como base de la enseñanza. Barcelona: Morata.

Tobon, S. 2007. Competencias en la educación superior. Bogotá: ECOE.

Unesco. 1994. La educación encierra un tesoro. Consulta en; http://www.unesco.org/ education/pdf/DELORS_S.PDF. (versión electrónica). 